Anaesthesist 2022 71 (Suppl 2):S241 https://doi.org/10.1007/s00101-022-01086-y Online publiziert: 12. Januar 2022 (c) The Author(s), under exclusive licence to Springer Medizin Verlag GmbH, ein Teil von Springer Nature 2022

\section{Erratum zu: Beweislastumkehr - Studien müssen zeigen, dass Trinken bis Abruf in den Operationssaal nicht sicher ist}

Anne Rüggeberg · Eike Nickel

Klinik für Anästhesiologie und Schmerztherapie, Helios Klinikum Emil von Behring, Berlin, Deutschland

\section{Erratum zu:}

Anaesthesist 2021

https://doi.org/10.1007/s00101-021-

01078-4

Aufgrund einer fehlerhaften Angabe im Text wurde der folgende Satz im Leserbrief aktualisiert: Walker et al. fanden bei 118.371 Kindern eine Inzidenz der Aspiration von $0,02 \%$. Alle Kinder mit Aspiration waren nüchtern und haben sich wiederkomplett erholt [9].

Wir bitten Sie die aktualisierte Version des Artikels zu berücksichtigen und den Fehler zu entschuldigen.

\section{Korrespondenzadresse}

\section{Dr. Anne Rüggeberg}

Klinik für Anästhesiologie und Schmerztherapie, Helios Klinikum Emil von Behring Walterhöferstr. 11, 14165 Berlin, Deutschland anne-rueggeberg@web.de 